\title{
The Relationships Among Sleep, Nutrition, and Obesity
}

\author{
Teresa Arora $^{1,2} \cdot$ Sopna Choudhury ${ }^{1,2} \cdot$ Shahrad Taheri ${ }^{1,2}$
}

Published online: 14 October 2015

(C) Springer International Publishing AG 2015

\begin{abstract}
Obesity is associated with an array of adverse physiological, psychological, and social consequences. Tackling obesity remains difficult, given the challenges of maintaining positive behavior changes. Obesity occurs from persistent positive energy (excessive energy intake with insufficient counteractive energy expenditure), but several biological, psychological, and environmental factors influence energy balance. It has recently emerged that sleep duration is an important factor that impinges on energy balance and could be a potential overlooked behavior predisposing to obesity. We highlight and review the recent evidence surrounding sleep in relation to obesity from epidemiological studies to experimental work.
\end{abstract}

Keywords Sleep duration $\cdot$ Sleep quality $\cdot$ Sleep loss $\cdot$ Sleep restriction $\cdot$ Circadian preference $\cdot$ Circadian misalignment . Obesity $\cdot$ Metabolism $\cdot$ Nutrition $\cdot$ Food intake $\cdot$ Energy balance $\cdot$ Appetite $\cdot$ Hunger

This article is part of the Topical Collection on Sleep Epidemiology

Shahrad Taheri

szt2004@qatar-med.cornell.edu

Teresa Arora

tea2006@qatar-med.cornell.edu

Sopna Choudhury

smc2009@qatar-med.cornell.edu

1 Department of Medicine and Clinical Research Core, Weill Cornell Medical College, Qatar Foundation, Education City, Room C008, PO Box 24144, Doha, Qatar

2 Department of Medicine and Clinical Research Core, Weill Cornell Medical College, New York, USA

\section{Introduction}

\section{The Problem of Obesity}

The prevalence of obesity has resulted in a global epidemic in recent times. Worldwide, in 2014, there were an estimated 1.9 billion overweight adults, 18 years and older, with over 600 million of these classed as obese [1]. In a large cohort of over 20,000 adult participants in the USA, the prevalence of overweight and obesity increased from 31.2 to $36.9 \%$ and from 10.2 to $27.7 \%$ from 1977 to 2009, respectively [2]. The obesity epidemic is affecting not only adults but also children, with the World Health Organization (WHO) estimating that in 2013, 42 million children younger than 5 years of age were either overweight or obese [1]. This is of a greater concern as childhood obesity tends to persist into adulthood and increases the risk and severity of obesity-associated diseases [3-6]. The WHO defines overweight and obesity as "abnormal or excessive fat accumulation that presents a risk to health" [1]. Body mass index (BMI; weight $\left.(\mathrm{kg}) /[\text { height }(\mathrm{m})]^{2}\right)$ is commonly used as a measure to classify overweight and obesity. In adults, the WHO classifies overweight as a BMI of greater than or equal to $25 \mathrm{~kg} / \mathrm{m}^{2}$ and obesity as a BMI of greater than or equal to $30 \mathrm{~kg} / \mathrm{m}^{2}$ although lower cutpoints have been recommended in several other populations [1].

Obesity is not only a significant challenge to those burdened with the disease and its complications but also has a serious impact upon health-care systems and society as a whole. Some of the many comorbidities associated with obesity include type 2 diabetes mellitus [7], cardiovascular disease (CVD), several cancers [3], and depression [8]. Obesity and overweight have also been associated with increased fasting glucose levels, a reflection of insulin insensitivity [9]. Key drivers to obesity comorbidities such as insulin insensitivity are changes in the array of adipocytokines released by adipocytes and chronic inflammation. 
Based on the first law of thermodynamics, weight gain occurs when the consumption of calories exceeds overall energy expenditure including physical activity. Excess energy is stored primarily in white adipose tissue as fat [10]. Major contributing factors to excess energy intake are high-fat calorie-dense foods and sugar-sweetened beverages combined with large portion size $[10,11]$. The quantity and nutritional composition of food ingested are influenced by many factors including biological, psychological, and environmental factors [12].

\section{Obesity and Sleep}

In recent years, while the prevalence of obesity has increased, several studies have reported a decline in sleep duration [13]. This suggests that there may be a relationship between obesity and sleep duration, which could be due to the opportunities and demands of modern society, use of media, socioeconomic status, work schedules, family dynamics, culture, and/or mental health. The prevalence of very short sleep $(<5 \mathrm{~h})$ and short sleep (5-6 h) in the USA increased from 1.7 to $2.4 \%$ and from 19.7 to $26.7 \%$ from 1977 to 2009 , respectively [2]. The prevalence for long sleep decreased from 11.6 to $7.8 \%$ during the same time period [2]. Data regarding secular trends in sleep duration, however, are controversial, as there are many deficiencies in the available data and not all reports confirm a reduction in sleep duration over time.

Sleep, or the lack of it, is increasingly recognized as a risk factor for obesity. Sleep is important as it plays a significant role in the restorative process of the body and its energy metabolism. In sleep, the body requires less energy to support brain function, sympathetic activity, breathing, and circulation are reduced; and the core body temperature is lower so there is a 20-30\% decrease in basal metabolic rate. The lack of sleep may therefore hinder these processes and cause adverse effects, such as weight gain and obesity.

Epidemiological studies have linked short sleep duration with the development of obesity and other metabolic disorders such as type 2 diabetes mellitus. Weight gain and obesity associated with short sleep duration have been attributed to increased ghrelin levels and decreased leptin levels. Ghrelin is a stomach-derived hormone that signals hunger to the hypothalamus to initiate food intake. Ghrelin levels are lower in obesity as a compensatory mechanism but are high in conditions associated with excess food intake such as Prader-Willi syndrome. Low ghrelin levels have also been observed post gastric bypass surgery, suggesting that it has a key role in reduction of appetite and weight loss after the procedure. Leptin is released by adipocytes to signal sufficient energy stores to the hypothalamus. In obesity, leptin levels are higher, suggesting the presence of leptin resistance, which has been shown to be due to defective leptin transport to the central nervous system. Thus, high leptin levels, as occurring in obesity, are not physiologically as important as low leptin levels which signal energy deficit triggering increased food intake. When leptin levels are low and ghrelin is high, this results in an increased appetite. These changes are observed after calorie deficit as occurring during dieting and may explain why weight loss can be so difficult. Low leptin and high ghrelin levels associated with short sleep can induce an increase of daily energy consumption, subsequent weight gain and obesity.

In adults, habitual short (sleeping $\leq 6 \mathrm{~h}$ per night) and long (sleeping $>9 \mathrm{~h}$ nightly) sleep durations have been recognized as behavioral risk factors for morbidity and mortality [14]. The association between short sleep duration and BMI has been reported by many epidemiological crosssectional and prospective studies. Studies have shown a Ushaped relationship between habitual sleep duration and body weight in adults. Data from 1024 volunteers from the Wisconsin Sleep Cohort Study highlighted that those who slept less than $8 \mathrm{~h}$ per night ( $74.4 \%$ of the sample) were more likely to be overweight [15]. The study also found that short sleep duration was associated with low leptin and high ghrelin levels. Independent of BMI, the study also observed $15.5 \%$ lower leptin for habitual sleep of $5 \mathrm{~h}$ versus $8 \mathrm{~h}$ and $14.9 \%$ higher ghrelin for nocturnal (polysomnographic) sleep of $5 \mathrm{~h}$ versus $8 \mathrm{~h}$ [15]. The changes in the levels of these hormones are equivalent to those observed with about a $1000 \mathrm{kcal}$ daily energy deficit. More recently, a study analyzed self-reported data from the National Health and Nutrition Examination Survey (NHANES) between 2005 and 2010. This study found an inverse linear relationship in the 13,742 study participants aged $\geq 20$ years (median age of 46 years). The short sleepers, defined as sleep duration of $\leq 6 \mathrm{~h}$, were heavier (approximately $1.7 \mathrm{~kg} / \mathrm{m}^{2}$, SE 0.4 ) and had a larger waist circumference $(3.4 \mathrm{~cm}, \mathrm{SE} 1.0)$ than long

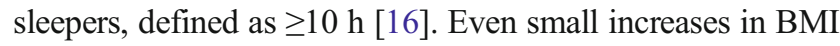
over time can be associated with increased risk of pre-diabetes and diabetes. Furthermore, such a change in BMI has a serious impact on health at a population level.

Interestingly, Nagai and colleagues found no association between sleep duration and risk of weight gain $\geq 5 \mathrm{~kg}$ ( $p$ for trend $=0.62$ ) and obesity in their Japanese participants when they examined the long-term association between sleep duration, weight gain, and obesity [17]. However, they did observe a significantly increased risk of $\geq 5 \mathrm{~kg}$ weight gain (odds ratio [OR] 1.36, $95 \%$ CI 1.09-1.70) in obese long sleepers ( $\geq 9 \mathrm{~h}$ of sleep). After acknowledging that this group was the eldest in the analysis (mean age of 61.9 years) and so may have included participants bedridden due to physical limitation, they further analyzed the data to exclude poor self-rated health, functional limitations, or history of disease. Even then, only obese long sleepers were found to be at significantly increased risk of weight gain (OR 1.41, $95 \%$ CI 1.04-1.92). 
An independent and inverse relationship was found more recently between sleep duration and visceral fat tissue accumulation. This was reported by Chaput and colleagues who carried out longitudinal analysis on 293 participants to investigate the relationship between change in sleep duration and long-term visceral adiposity alteration in adults [18]. They found that over the 6-year follow-up period, on average, participants gained $19.2 \pm 37.3 \mathrm{~cm}^{2}$ in visceral fat tissue. They observed an independent association with $6 \mathrm{~cm}^{2}$ fewer visceral fat tissue gain over the follow-up period and a change in sleep duration from $\leq 6$ to $7-8 \mathrm{~h} /$ day.

Short sleep duration, resulting in insufficient sleep, can cause neuroendocrine, metabolic, and behavioral adaptations. This, in combination with the abundance of freely available energy dense foods, can cause a greater energy imbalance and lead to weight gain and resultant obesity. Dietary and sleep behaviors have been a field of growing research interest. Using the NHANES data $(n=14,992)$, short sleepers $(\leq 6 \mathrm{~h}$ of sleep) were found to not only to eat earlier in the day but also ate later in the day [19]. Most studies support the concept that restricting sleep increases food intake. However, more eating events were not reported in this NHANES analysis. Interestingly though, this analysis showed that a smaller proportion of short sleepers, compared to average sleepers (7-8 $\mathrm{h}$ of sleep), reported eating breakfast and dinner $(p<0.04)$. A higher percentage of short sleepers who reported having breakfast also reported having a snack (defined as eating episodes that were not main meals) before breakfast $(p \leq 0.004)$.

There are several deficiencies in population-based studies that need to be considered. The majority of the available studies have been cross-sectional with many not originally designed to examine the impact of sleep on health. There has been a lack of use of objective measures to determine sleep duration and quality in naturalistic environments. Often, sleep has been assessed by only a single question. Nevertheless, the body of evidence is highly compelling and is also supported by experimental human sleep laboratory and animal studies, which have identified some of the potential mechanisms.

While the epidemiological and prospective evidence continues to support the short sleep-obesity association, the focus of attention has increasingly switched to mechanistic explanations, which include energy intake/expenditure and feeding behaviors in response to sleep loss. Diet is difficult to quantify in large samples and usually relies upon subjective reports, which are prone to multiple biases/inaccuracies. Experimental evidence in tightly controlled settings has revealed important explanations.

\section{Sleep Loss and Excess Energy Intake: Experimental Evidence}

Detailed experimental work has provided a number of mechanistic clues concerning the relationship between sleep, metabolic disturbance, and obesity. The effects of sleep loss upon dietary behaviors has been a recent topic of interest. A number of studies offer explanations regarding how sleep loss can result in weight gain and obesity. Spaeth and colleagues have performed the largest randomized sleep restriction study to date, recruiting 225 adults aged $22-50$ years [20]. Participants were randomized to either five nights of 4-h time in bed (0400-0800 hours) or the control condition of 10$\mathrm{h}$ time in bed (2200-0800 hours). Those in the 5-day sleep restriction group gained $0.86 \mathrm{~kg}$ more weight compared to controls $(p<0.007)$. Furthermore, sleep-restricted individuals consumed $30 \%$ additional calories per day compared to controls $(p=0.003)$. Specifically, the extra calories consumed were a result of more meals and calories during evening/ early hours (2200-0359 hours). Moreover, calories consumed from fat between 2200 and 0359 hours was approximately 5 $\%$ higher compared to daytime (0800-1459 hours) and over 3 $\%$ more compared to $1500-2159$ hours, $p<0.05$. The same group, using the same study protocol, investigated differences between ethnic groups for energy intake with sleep restriction versus without. There was no evidence for differences between African-Americans and Caucasians for calorie intake. The findings, however, remained consistent for increased calorie consumption (about $24 \%, p<0.001$ ) and fat intake $(>1 \%$, $p=0.024$ ) following sleep restriction in the total sample [21].

Portion size selection/consumption is one possible explanation for increased calorie intake observed in relation to acute total sleep deprivation compared to healthy sleep $(8 \mathrm{~h})$. This was recently explored by Hogenkamp and colleagues in 16 young, healthy men who were recruited to a randomized crossover study with two conditions: one night of total sleep deprivation (TSD) and one night of 8 -h time in bed (monitored with polysomnography, PSG) separated by a 28-day washout period [22]. Subsequent to each condition, participants rated their hunger using a $10-\mathrm{cm}$ visual analogue scale and completed a "portion size task." This task included presentation of images on a laptop (seven meals and six snacks). Participants were asked to imagine that they could have the food that appeared in the image and identify portion size using arrow keys on the keyboard to either increase or decrease the portion size until it represented what they desired. The authors calculated the energy value for each rating, and the participants completed the task in both a fasted and non-fasted state. Subjective hunger ratings were confirmed to be higher following TSD compared to recommended sleep duration of $8 \mathrm{~h}$, supporting previous findings. For all food items (meals and snacks) combined, average portion size when in a fasted state and sleep deprived (482 kcal) was significantly elevated compared to the 8 -h sleep condition ( $422 \mathrm{kcal}$ ), $p=0.02$. No significant differences were reported when the food items were stratified by meal/snack and fasted between sleep conditions. It is of interest that when participants were in a nonfasted state, there was an overall significant effect of 
combined food items when sleep deprived versus rested (399 and $356 \mathrm{kcal}$, respectively, $p<0.01$ ) as well as snacks (364 and $313 \mathrm{kcal}$, respectively, $p=0.02$ ). No differences were observed for meal items between sleep conditions in the non-fasted state. Similar findings, by the same group, have been reported in relation to food purchasing when provided with a fixed budget and TSD [23]. Limitations of the study are that TSD is not representative of societal sleep behaviors where sleep quantity is reduced or insufficient in a proportion of the population. Also, partial sleep loss that commonly occurs is likely to result in an accumulated sleep debt over time that will have an impact on metabolic regulation, cognitive function, and decision-making. Furthermore, desired portion sizes provide only an indication of appetite for the food item presented but do not quantify actual consumption. Finally, the results may not be generalizable to other populations (children, older adults, women) although the findings appear consistent in small samples of children [24] and adolescents [25, 26] with the effect of gender being recently explored.

Gender differences for metabolic regulation have been demonstrated in response to sleep restriction. Male and female volunteers $(n=27)$ with healthy weights aged $30-45$ years, recruited to a randomized crossover study, were exposed to two sleep conditions (three nights of 4 or $9 \mathrm{~h}$ in bed) and provided with a controlled diet with fixed timings for meals [27]. The levels of two opposing gut hormones, ghrelin and glucagon-like peptide-1 (GLP-1), were altered in men but not in women following sleep restriction. Ghrelin, associated with hunger levels, increased with sleep restriction compared to controls. Conversely, GLP-1, associated with satiety and pancreatic beta cell insulin secretion, was reduced following sleep restriction. In combination, persistent metabolic hormone changes may promote the onset of type 2 diabetes mellitus and obesity through surplus energy intake. Based on this study, different mechanisms are likely according to gender although the underlying reasons for this are not yet clear.

\section{The Psychology of Sleep and Over-feeding}

While these recent findings that suggest a relationship between sleep loss and food intake beyond individual requirements may be partially explained by alterations in energy metabolism, psychological processes have also been implicated. For example, sleep loss has been previously shown to impair cognitive function [28] in a dose-dependent manner. Sleep loss in relation to decision-making and impulsivity has also been documented, although there is limited evidence regarding food selection/intake. Preliminary evidence has emerged from a recent study of 14 young (mean age of 22 years), healthy men [29]. In a randomized crossover study, participants underwent $24 \mathrm{~h}$ of total sleep deprivation (TSD) and one night of 8.5 -h time in bed while being monitored by polysomnography (PSG), separated by a 4 -week washout period. The morning after each condition, participants selfreported hunger levels and fasting blood samples were drawn for assessment of glucose concentration, followed by a cognitive task to determine inhibition (go/no-go task: the participant was presented with a series of non-food- and food-related words and instructed to press a button only when a nonfood-related word was viewed) with the number of errors serving as the primary outcome measure. Main findings of this study included a $56 \%$ increased number of errors on the inhibition task following TSD compared to the normal sleep condition $(p<0.05)$. Consistent with other research findings, subjective hunger (measured using a visual analogue scale) significantly increased from $2.7 \mathrm{~cm}$ following 8.5 -h time in bed to $6.3 \mathrm{~cm}$ following TSD. Contrary to other studies [30], no significant differences were observed between the two conditions for glucose levels. While TSD does not reflect societal sleep behaviors, this initial evidence has revealed one possible mechanistic explanation of how sleep loss can skew energy balance.

Further advances have been recently made which support neuronal networks and responses to food stimuli following partial sleep loss. A study of 25 healthy weight men $(n=13)$ and women $(n=12)$ was randomized to undertake five nights of either 4 or $9 \mathrm{~h}$ time in bed [31]. At the end of the sleep condition, participants underwent functional magnetic resonance imaging (fMRI) in a fasted state and were presented with images of healthy and unhealthy food types. Neuronal response networks were assessed and compared between healthy and unhealthy food stimuli. Heightened activation of specific neuronal regions, associated with reward, was observed in response to unhealthy food images compared to healthy stimuli in participants who were sleep restricted. Specifically, the right insula had greater activation for unhealthy foods following sleep restriction, a brain region that mediates energy homeostasis and pleasure-seeking behaviors. These preliminary findings have aided a better mechanistic understanding of how sleep loss can affect neuronal networks and cognitive processes. More research in this area is, however, required to ensure full understanding of the processes involved in feeding behaviors in response to sleep loss. Moreover, these studies could be repeated to investigate the potential effect of energy expenditure on neuronal processes following manipulation of sleep loss.

\section{Sleep Loss and Insufficient Energy Expenditure: Experimental Evidence}

Positive energy occurs not only from excess energy intake but also from a lack of compensatory energy expenditure. While the evidence surrounding excess energy intake from experimental sleep manipulation studies continues to support a link between positive energy balance, it is important to consider both sides of the energy balance equation. Excess energy 
intake is acceptable if energy expenditure can counteract it and has been the recent focus of research attempting to complete our mechanistic understanding of the sleep-feeding-obesity relationship. Of the few studies that have examined sleep restriction and energy expenditure in adults, the findings are varied. One study confirmed increased calorie intake with sleep restriction (two thirds of habitual sleep duration) but found no change in energy expenditure [32]. Of note, energy expenditure was measured through utilization of accelerometry, which has been used and validated to estimate physical activity level rather than energy expenditure per se. Markwald and colleagues reported opposing evidence of energy expenditure measured by wholeroom calorimetry [33]. An increase in total daily energy expenditure (about $5 \%$ ) was observed at the end of a five-night period of sleep restriction, designed to represent a typical working week where individuals have 5 -h time in bed, compared to adequate sleep (9 h). Consistent with previous studies [34, 35], increased energy intake was observed in response to sleep restriction but the increase in energy expenditure was insufficient, resulting in positive energy and weight gain $(0.82 \mathrm{~kg})$ over just 5 days/nights.

The effect of disrupted sleep upon energy expenditure has also been explored in experimental studies. Using a randomized crossover design, Hursel and colleagues subjected 15 healthy, young men to two conditions: two nights of $8 \mathrm{~h}$ of undisturbed sleep and $8 \mathrm{~h}$ of disturbed sleep where participants received acoustic stimuli every hour while being monitored with PSG [36]. Energy expenditure was measured using a respiratory chamber where participants were housed throughout each sleep condition. Sleep quality was quantified using slow-wave sleep (SWS), rapid eye movement (REM) sleep, and total sleep time to derive sleep quality percentage. An inverse relationship was observed between total energy expenditure and sleep quality, where $r=-0.57, p=0.03$. A possible explanation for this is that wakefulness, occurring from disrupted sleep, will enhance movement and therefore increase energy output. Furthermore, higher activity levels may promote better sleep quality.

Extended wakefulness may promote physical inactivity and provide increased opportunity to feed. Sleep loss clearly results in increases in energy intake, possibly to combat reduced energy levels arising from sleep deprivation. Sleep deprivation per se, results in shifted sleep-wake timings and thus challenges circadian rhythmicity, an area recently investigated in relation to energy balance and metabolic disturbance.

\section{The Effects of a Challenged Circadian Clock on Metabolic Health}

Wakefulness during night hours places a challenge upon the intrinsic biological (circadian) rhythm. Wakefulness when the circadian clock encourages sleep has been shown to result in serious, yet preventable, adverse health consequences. Shift workers serve as a comparable experimental model, and research has consistently verified a constellation of negative outcomes including elevated body mass index (BMI), deranged glucose metabolism, and increased prevalence of type 2 diabetes mellitus, cardiovascular disease, and metabolic syndrome $[37,38]$. Night shift work is an extreme example of circadian challenge, and recent experimental work by McHill and colleagues, which mimicked night shift work, supports the theory of disrupted energy metabolism with circadian misalignment. The study, which recruited 14 young, healthy adults (eight females) to a 6-day inpatient stay and mimicked shift work, observed a number of significant findings [39]. The volunteers were monitored with wrist actigraphy for 1-week prior to laboratory attendance to verify sleep-wake patterns, sleep duration (about $8 \mathrm{~h}$ ), and light exposure. Sleep opportunity was experimentally manipulated after day 3 of the inpatient stay when volunteers were awakened at their usual wake time, provided with a 2-h nap opportunity before the first simulated night shift (awake all night) and then given an 8-h sleep opportunity during day 4 which commenced $1-\mathrm{h}$ after their usual wake time. A further two night shifts were scheduled with an 8-h daytime sleep opportunity. Multiple outcome measures were ascertained including energy expenditure and macronutrient utilization determined by whole-room indirect calorimetry and 24-h urine sampling. A series of blood samples were also obtained to determine levels of leptin, ghrelin, peptide tyrosine-tyrosine (PYY; a gut-derived satiety hormone), and pineal-derived melatonin. Subjective hunger was measured every $2 \mathrm{~h}$ using a visual analogue scale, and PSG was utilized to assess multiple sleep characteristics. Three meals per day were provided to meet energy balance needs, although there was no indication of what the meals contained or how individual energy requirements were determined. The main finding was the significant reduction in daily total energy expenditure, albeit minimal (about $3 \%$ ). Protein and carbohydrate utilization was significantly reduced on the second night shift only. Hunger levels decreased despite a significant reduction from baseline in leptin (about $41 \%$ ) and PYY (about $11 \%$ ) levels, which signal satiety through hypothalamic pathways. These findings have enhanced our understanding of energy expenditure in response to extreme circadian misalignment. However, circadian preference is not usually this extreme, with the exception of some sleep disorders (advance sleep phase delay, delayed sleep phase syndrome).

Circadian preference (chronotype), which is either late (night owls, usually prevalent in adolescents) or early (early bird/larks, more commonly observed in older adults), is likely to result in altered sleep-wake timings and has been the recent focus of study in relation to energy metabolism and energy intake. Our knowledge concerning sleep restriction and its relationship with energy intake has recently been extended with some groups exploring the association between sleep timing (a reflection of circadian preference) and food selection. A recent study assessed chronotype (verified by wrist actigraphy), BMI, and self-reported eating habits of 511 
young adolescents [40]. Compared to morning types, evening chronotype was positively associated with BMI $z$-score $(\beta=$ $0.51, p<0.01)$. Poorer dietary habits, including increased frequency of eating unhealthy snacks and night-time caffeine consumption as well as inadequate daily intake of fruit and vegetables $(p \leq 0.01)$, were also observed in those with a later circadian preference. While it can be argued that circadian phase delay is more common in adolescents, there are consistent data in adults for metabolic disruption [41] as well as type 2 diabetes mellitus [42] and the metabolic syndrome [42].

From an evolutionary perspective, sleep timing per se, rather than sleep duration/loss, may be an important factor for optimum metabolic regulation and energy balance. For example, one study collected information on sleep-wake timings (sleep diary) and assessed delayed sleep timings relative to physical activity (waist-worn actigraphy) in 22 adults. After adjusting for sleep length, both later sleep $(p=0.013)$ and wake times $(p=$ 0.005 ) were significantly associated with less time performing moderate-to-vigorous physical activity and increased sedentariness [43]. Given that both sleep loss and late chronotype have been linked to obesity and metabolic disturbance, it would be feasible to assume that if the two were combined, a greater effect would be observed. This was recently explored under tightly controlled laboratory conditions, subjecting 24 participants to 3 weeks of reduced sleep $(5.6 \mathrm{~h})$ paired with circadian disruption [44]. Standardized meals were provided, and regular blood sampling was performed to investigate levels of numerous metabolic hormones. While all participants were free of metabolic disease at baseline, during the sleep manipulation, three volunteers qualified for pre-diabetes based on post-prandial glucose levels. The levels of ghrelin were significantly increased during the sleep loss/circadian misalignment phase $(p<0.05)$, and leptin was lower $(p=0.05)$ compared to baseline levels. While normalized sleep was permitted for nine nights after sleep manipulation and those with pre-diabetes reverted back to healthy glucose levels, to disentangle the relationship, future studies could randomize individuals to either (1) continue with sleep loss and normalize the circadian rhythm or (2) continue with circadian misalignment with a recovery sleep opportunity. Identifying which sleep parameter has the greatest effect will better inform future strategies for targeting the treatment of those with metabolic disease. There are some preliminary data supporting improved weight loss outcomes in early chronotypes with earlier meal timings [20]. Further study is required, but combining lifestyle changes such as going to bed earlier and eating meals earlier may be simple yet effective behavior changes that are relatively easy to adhere to.

\section{Napping: Good or Bad?}

Sleep deprivation results in a higher propensity to nap during the day, although this behavior may also occur in the absence of sleep problems. The consequences/benefits of napping are unclear with heterogeneous findings [45-47]. Habitual, frequent napping has been previously associated with an increased risk of type 2 diabetes mellitus after adjustment for multiple sleep factors that indicate insufficient nocturnal sleep [46]. Conversely, others have shown improvements in cognitive performance with napping, subsequent to acute total sleep loss in a laboratory setting [48].

It is not yet known if providing a nap opportunity can help to alleviate adverse metabolic hormone alterations that promote hunger, appetite, and satiety, in response to accumulated sleep debt. Furthermore, napping may help to improve subsequent hunger, appetite, and satiety levels observed with sleep insufficiency. Napping (2-h opportunity) in response to acute total sleep deprivation has been previously explored [49]. Contrary to previous evidence [29], no association was found between subjective hunger and $24 \mathrm{~h}$ of total sleep deprivation. Participants randomized to a 2-h nap opportunity condition did not report significant alterations in subjective hunger ratings. While this initial evidence is not promising, further investigation in this area is needed. Total sleep deprivation is unrepresentative of societal sleep behaviors; thus, protocols intended for chronic partial sleep restriction with and without napping opportunities are required. This will help to assess if napping is a potential approach for regulating energy intake, appetite, hunger, and metabolic hormones associated with feeding behaviors.

A consistent recommendation for improving health outcomes in short sleepers is to develop and apply a sleep extension intervention to improve nocturnal sleep. It may, however, be possible to achieve the same outcomes with napping as a means of overcoming hunger and over-eating, following inadequate sleep. This has not yet been examined but may provide an avenue for future research.

\section{Conclusions}

There is some preliminary evidence that extending sleep quantity in overweight, short sleepers is possible, with beneficial effects on energy balance [50] but additional studies are required. It is clear from emerging evidence that sleep plays a major role in the regulation of energy metabolism. Sleep loss clearly results in metabolic derangements, changes in appetite, hunger, and feeding, all of which may lead to surplus energy. While one function of sleep is to preserve energy, it is assumed that sleep loss, from additional wakefulness, results in increased energy expenditure although this has not been consistently confirmed. Conflicting suggestions include the proposal that inadequate sleep results in tiredness and may, in turn, lead to reduced motivation to engage in physical activity. Detailed evidence is needed for energy expenditure in response to sleep loss and circadian desynchronization. Experimental napping to reduce sleep debt may or may not improve energy metabolism, although this is still to be established. 
Acknowledgments All authors are funded by the Weill Cornell Medical College (Qatar) Biomedical Research Program (BMRP) supported by Qatar Foundation.

\section{Compliance with Ethical Standards}

Conflict of Interest Teresa Arora, Sopna Choudhury, and Shahrad Taheri declare that they have no conflict of interest.

Human and Animal Rights and Informed Consent This article does not contain any studies with human or animal subjects performed by any of the authors.

\section{References}

1. WHO Obesity and overweight [Internet]. WHO. [cited 5 Jul 2015]. Available from: http://www.who.int/mediacentre/factsheets/fs311/ en/. Access date 12 Oct 2015.

2. Jean-Louis G, Williams NJ, Sarpong D, Pandey A, Youngstedt S, Zizi F, et al. Associations between inadequate sleep and obesity in the US adult population: analysis of the national health interview survey (1977-2009). BMC Public Health. 2014;14(1):290.

3. Guo SS, Chumlea WC. Tracking of body mass index in children in relation to overweight in adulthood. Am J Clin Nutr. 1999;70(1): $145 \mathrm{~S}-8 \mathrm{~S}$.

4. Freedman DS, Khan LK, Serdula MK, Dietz WH, Srinivasan SR, Berenson GS. The relation of childhood BMI to adult adiposity: the Bogalusa Heart Study. Pediatrics. 2005;115(1):22-7.

5. Freedman DS, Khan LK, Dietz WH, Srinivasan SR, Berenson GS. Relationship of childhood obesity to coronary heart disease risk factors in adulthood: the Bogalusa Heart Study. Pediatrics. 2001;108(3):712-8.

6. Freedman DS, Wang J, Thornton JC, Mei Z, Sopher AB, Pierson $\mathrm{RN}$, et al. Classification of body fatness by body mass index-forage categories among children. Arch Pediatr Adolesc Med. 2009;163(9):805-11.

7. Huerta JM, Tormo M-J, Chirlaque M-D, Gavrila D, Amiano P, Arriola L, et al. Risk of type 2 diabetes according to traditional and emerging anthropometric indices in Spain, a Mediterranean country with high prevalence of obesity: results from a large-scale prospective cohort study. BMC Endocr Disord. 2013;13(1):7.

8. Schneider B, Lukaschek K, Baumert J, Meisinger C, Erazo N, Ladwig K-H. Living alone, obesity, and smoking increase risk for suicide independently of depressive mood findings from the population-based MONICA/KORA Augsburg cohort study. J Affect Disord. 2014;152-154:416-21.

9. Chen L, Chen R, Wang H, Liang F. Mechanisms linking inflammation to insulin resistance. Int J Endocrinol. 2015;2015:1-9.

10. Chugh PK, Sharma S. Recent advances in the pathophysiology and pharmacological treatment of obesity. J Clin Pharm Ther. 2012;37(5):525-35.

11. Muñoz-Pareja M, Guallar-Castillón P, Mesas AE, López-García E, Rodríguez-Artalejo F. Obesity-related eating behaviors are associated with higher food energy density and higher consumption of sugary and alcoholic beverages: a cross-sectional study. PLoS One. 2013;8(10):e77137.

12. Skolnik NS, Ryan DH. Pathophysiology, epidemiology, and assessment of obesity in adults. J Fam Pract. 2014;63(7 Suppl):S3-10.

13. Bin YS, Marshall NS, Glozier N. Secular trends in adult sleep duration: a systematic review. Sleep Med Rev. 2012;16(3):223-30.

14. Grandner MA, Sands-Lincoln MR, Pak VM, Garland SN. Sleep duration, cardiovascular disease, and proinflammatory biomarkers. Nat Sci Sleep. 2013;5:93-107.
15. Taheri S, Lin L, Austin D, Young T, Mignot E. Short sleep duration is associated with reduced leptin, elevated ghrelin, and increased body mass index. PLoS Med. 2004;1(3):e62.

16. Ford ES, Li C, Wheaton AG, Chapman DP, Perry GS, Croft JB. Sleep duration and body mass index and waist circumference among US adults: sleep duration and anthropometric parameters. Obesity. 2014;22(2):598-607.

17. Nagai M, Tomata Y, Watanabe T, Kakizaki M, Tsuji I. Association between sleep duration, weight gain, and obesity for long period. Sleep Med. 2013;14(2):206-10.

18. Chaput J-P, Bouchard C, Tremblay A. Change in sleep duration and visceral fat accumulation over 6 years in adults. Obes Silver Spring Md. 2014;22(5):E9-12.

19. Kant AK, Graubard BI. Association of self-reported sleep duration with eating behaviors of American adults: NHANES 2005-2010. Am J Clin Nutr. 2014;100(3):938-47.

20. Garaulet M, Gómez-Abellán P, Alburquerque-Béjar JJ, Lee Y-C, Ordovás JM, Scheer FA. Timing of food intake predicts weight loss effectiveness. Int J Obes 2005. 2013;37(4):604-11.

21. Spaeth AM, Dinges DF, Goel N. Sex and race differences in caloric intake during sleep restriction in healthy adults. Am J Clin Nutr. 2014;100(2):559-66.

22. Hogenkamp PS, Nilsson E, Nilsson VC, Chapman CD, Vogel H, Lundberg LS, et al. Acute sleep deprivation increases portion size and affects food choice in young men. Psychoneuroendocrinology. 2013;38(9):1668-74.

23. Chapman $\mathrm{CD}$, Nilsson $\mathrm{EK}$, Nilsson $\mathrm{VC}$, Cedernaes J, Rångtell $\mathrm{FH}$, Vogel $\mathrm{H}$, et al. Acute sleep deprivation increases food purchasing in men. Obes Silver Spring Md. 2013;21(12):E555-60.

24. Hart CN, Carskadon MA, Considine RV, Fava JL, Lawton J, Raynor HA, et al. Changes in children's sleep duration on food intake, weight, and leptin. Pediatrics. 2013;132(6):e1473-80.

25. Beebe DW, Simon S, Summer S, Hemmer S, Strotman D, Dolan LM. Dietary intake following experimentally restricted sleep in adolescents. Sleep. 2013;36(6):827-34.

26. Simon SL, Field J, Miller LE, DiFrancesco M, Beebe DW. Sweet/ dessert foods are more appealing to adolescents after sleep restriction. PLoS One. 2015;10(2):e0115434.

27. St-Onge M-P, O'Keeffe M, Roberts AL, RoyChoudhury A, Laferrère B. Short sleep duration, glucose dysregulation and hormonal regulation of appetite in men and women. Sleep. 2012;35(11):1503-10.

28. Van Dongen HPA, Maislin G, Mullington JM, Dinges DF. The cumulative cost of additional wakefulness: dose-response effects on neurobehavioral functions and sleep physiology from chronic sleep restriction and total sleep deprivation. Sleep. 2003;26(2):11726.

29. Cedernaes J, Brandell J, Ros O, Broman J-E, Hogenkamp PS, Schiöth HB, et al. Increased impulsivity in response to food cues after sleep loss in healthy young men. Obes Silver Spring Md. 2014;22(8):1786-91.

30. Gailliot MT, Baumeister RF. The physiology of willpower: linking blood glucose to self-control. Personal Soc Psychol Rev. 2007;11(4):303-27.

31. St-Onge M-P, Wolfe S, Sy M, Shechter A, Hirsch J. Sleep restriction increases the neuronal response to unhealthy food in normalweight individuals. Int J Obes 2005. 2014;38(3):411-6.

32. Calvin AD, Carter RE, Adachi T, Macedo PG, Albuquerque FN, van der Walt $\mathrm{C}$, et al. Effects of experimental sleep restriction on caloric intake and activity energy expenditure. Chest. 2013;144(1): 79-86.

33. Markwald RR, Melanson EL, Smith MR, Higgins J, Perreault L, Eckel RH, et al. Impact of insufficient sleep on total daily energy expenditure, food intake, and weight gain. Proc Natl Acad Sci U S A. 2013;110(14):5695-700. 
34. Jung CM, Melanson EL, Frydendall EJ, Perreault L, Eckel RH, Wright KP. Energy expenditure during sleep, sleep deprivation and sleep following sleep deprivation in adult humans. J Physiol. 2011;589(Pt 1):235-44.

35. Spaeth AM, Dinges DF, Goel N. Effects of experimental sleep restriction on weight gain, caloric intake, and meal timing in healthy adults. Sleep. 2013;36(7):981-90.

36. Hursel R, Gonnissen HKJ, Rutters F, Martens EAP, WesterterpPlantenga MS. Disadvantageous shift in energy balance is primarily expressed in high-quality sleepers after a decline in quality sleep because of disturbance. Am J Clin Nutr. 2013;98(2):367-73.

37. Ulhôa MA, Marqueze EC, Burgos LGA, Moreno CRC. Shift work and endocrine disorders. Int J Endocrinol. 2015;2015:826249.

38. Brum MCB, Filho FFD, Schnorr CC, Bottega GB, Rodrigues TC. Shift work and its association with metabolic disorders. Diabetol Metab Syndr. 2015;7:45.

39. McHill AW, Melanson EL, Higgins J, Connick E, Moehlman TM, Stothard ER, et al. Impact of circadian misalignment on energy metabolism during simulated nightshift work. Proc Natl Acad Sci U S A. 2014;111(48):17302-7.

40. Arora T, Taheri S. Associations among late chronotype, body mass index and dietary behaviors in young adolescents. Int J Obes 2005. 2015;39(1):39-44.

41. Reutrakul S, Hood MM, Crowley SJ, Morgan MK, Teodori M, Knutson KL, et al. Chronotype is independently associated with glycemic control in type 2 diabetes. Diabetes Care. 2013;36(9):2523-9.

42. Yu JH, Yun C-H, Ahn JH, Suh S, Cho HJ, Lee SK, et al. Evening chronotype is associated with metabolic disorders and body composition in middle-aged adults. J Clin Endocrinol Metab. 2015;100(4):1494-502.
43. Shechter A, St-Onge M-P. Delayed sleep timing is associated with low levels of free-living physical activity in normal sleeping adults. Sleep Med. 2014;15(12):1586-9.

44. Buxton OM, Cain SW, O'Connor SP, Porter JH, Duffy JF, Wang W, et al. Adverse metabolic consequences in humans of prolonged sleep restriction combined with circadian disruption. Sci Transl Med. 2012;4(129):129ra43.

45. Naska A, Oikonomou E, Trichopoulou A, Psaltopoulou T, Trichopoulos D. Siesta in healthy adults and coronary mortality in the general population. Arch Intern Med. 2007;167(3):296-301.

46. Lam K-BH, Jiang CQ, Thomas GN, Arora T, Zhang WS, Taheri S, et al. Napping is associated with increased risk of type 2 diabetes: the Guangzhou Biobank Cohort Study. Sleep. 2010;33(3):402-7.

47. Fang W, Li Z, Wu L, Cao Z, Liang Y, Yang H, et al. Longer habitual afternoon napping is associated with a higher risk for impaired fasting plasma glucose and diabetes mellitus in older adults: results from the Dongfeng-Tongji cohort of retired workers. Sleep Med. 2013;14(10):950-4.

48. Vgontzas AN, Pejovic S, Zoumakis E, Lin HM, Bixler EO, Basta $\mathrm{M}$, et al. Daytime napping after a night of sleep loss decreases sleepiness, improves performance, and causes beneficial changes in cortisol and interleukin-6 secretion. Am J Physiol Endocrinol Metab. 2007;292(1):E253-61.

49. Pejovic S, Vgontzas AN, Basta M, Tsaoussoglou M, Zoumakis E, Vgontzas A, et al. Leptin and hunger levels in young healthy adults after one night of sleep loss. J Sleep Res. 2010;19(4):552-8.

50. Tasali E, Chapotot F, Wroblewski K, Schoeller D. The effects of extended bedtimes on sleep duration and food desire in overweight young adults: a home-based intervention. Appetite. 2014;80:220-4. 